BNL- -46415

DE91 015845

Theory of the Tune Shift Due to Linear Coupling

\author{
G. Parzen
}

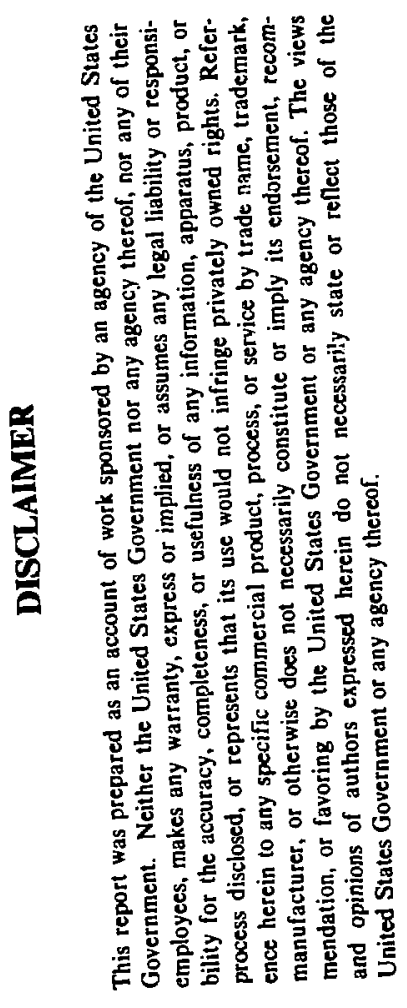

July 1991

RHIC PRO JECT

JUL. 51991

Brookhaven National Laboratory

Associated Universities, Inc.

Upton, NY 11973

Under Contract No. DE-AC02-76CH00016 with the

UNITED STATES DEPARTMENT OF ENERGY

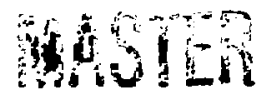




\title{
THEORY OF THE TUNE SHIFT DUE TO LINEAR COUPLING
}

\author{
G. Parzen \\ Brookhaven National Laboratory \\ July 1991
}

\section{Introduction}

The presence of skew quadrupole fields will linearly couple the $x$ and $y$ motions. The $x$ and $y$ motion can then be written as the sum of two normal modes ${ }^{1}$ which have the tunes $\nu_{1}$ and $\nu_{2}$ which are different from the tune, $\nu_{x}, \nu_{y}$, in the absence of the skew quadrupole fields. New beta functions, $\beta_{1}$ and $\beta_{2}$, can be defined ${ }^{2}$ which are the beta functions of the normal modes and which are different from $\beta_{x}$ and $\beta_{y}$, the beta functions of the unperturbed accelerator.

This paper presents analytical perturbation theory results for $\nu_{1}, \nu_{2}$. The results for $\nu_{1}, \nu_{2}$ are first found correct to lowest order in the skew quadrupole fields. The results for $\nu_{1}, \nu_{2}$ are then carried one step further to include the next higher order terms in the skew quadrupole fields. Results for $\beta_{1}, \beta_{2}$ will be given in a future paper.

These analytical results show that for the higher order shift in tune the important harmonics of the skew quadrupole field are the harmonics near $\nu_{x}+\nu_{y}$. However the harmonics closest to $\nu_{x}+\nu_{y}$ do not contribute to the higher order tune splitting, $\left|\nu_{1}-\nu_{2}\right|$, as they shift $\nu_{1}$ and $\nu_{2}$ about equally. This results in a lack of a dominant harmonic for the higher order contribution of $\left|\nu_{1}-\nu_{2}\right|$, which complicates the understanding and correction ${ }^{3}$ of the higher order contribution to $\left|\nu_{1}-\nu_{1}\right|$.

Analytical results are found for the residual tune splitting which is the $\left|\nu_{1}-\nu_{2}\right|$ that remains after the driving term of the nearby difference resonance has been corrected. 


\section{Lowest Order Solution for the Motion and the Tune}

The equations of motion can be written as

$$
\begin{aligned}
\left(\frac{d^{2}}{d \theta_{x}^{2}}+\nu_{x}^{2}\right) \eta_{x} & =b_{x}(s) \eta_{y} \\
\left(\frac{d^{2}}{d \theta_{y}^{2}}+\nu_{y}^{2}\right) \eta_{y} & =b_{y}(s) \eta_{x} \\
x & =\beta_{x}^{\frac{1}{2}} \eta_{x}, y=\beta_{y}^{\frac{1}{2}} \eta_{y} \\
\theta_{x} & =\int d s\left(1 / \nu_{x} \beta_{x}\right)=\psi_{x} / \nu_{x} \\
\theta_{y} & =\int d s\left(1 / \nu_{y} \beta_{y}\right)=\psi_{y} / \nu_{y} \\
b_{x}(s) & =\nu_{x}^{2} \beta_{x}\left(\beta_{x} \beta_{y}\right)^{1 / 2}\left(a_{1} / \rho\right) \\
b_{y}(s) & =\nu_{y}^{2} \beta_{y}\left(\beta_{x} \beta_{y}\right)^{1 / 2}\left(a_{1} / \rho\right) .
\end{aligned}
$$

The skew quadrupole field is described by $a_{1}(s)$. On the median plane, the field $B_{x}$ is given by

$$
B_{x}=-B_{0} a_{1} x
$$

where $B_{0}$ is the main dipole field. $\rho$ is the radius of curvature in the main dipole.

To simplify the solutions of Eq. (2.1), we introduce $\zeta_{x}$ and $\zeta_{y}$ such that

$$
\begin{aligned}
& \eta_{x}=\zeta_{x}+\text { c.c. } \\
& \eta_{y}=\zeta_{y}+\text { c.c. }
\end{aligned}
$$

$\zeta_{x}$ and $\zeta_{y}$ also satisfy Eq. (2.1). In addition, when $\alpha_{1}=0$, the solution for $\zeta_{x}, \zeta_{y}$ is

$$
\begin{aligned}
& \zeta_{x}=A \exp \left(i \nu_{x} \theta_{x}\right) \\
& \zeta_{y}=B \exp \left(i \nu_{y} \theta_{y}\right)
\end{aligned}
$$

We are looking for a solution of Eq. (2.1) which is valid when $\nu_{x}, \nu_{y}$ are close to the coupling resonance $\nu_{x}-\nu_{y}=p, p$ being some integer. The solution for $\zeta_{x}, \zeta_{y}$ will be assumed to have the form

$$
\begin{aligned}
& \zeta_{x}=A_{s} \exp \left(i \nu_{x, s} \theta_{x}\right)+\sum_{r \neq s} A_{r} \exp \left(i \nu_{x, r} \theta_{x}\right) \\
& \zeta_{y}=B_{s} \exp \left(i \nu_{y, s} \theta_{x}\right)+\sum_{r \neq s} B_{r} \exp \left(i \nu_{y, r} \theta_{y}\right) . \\
& \nu_{x, s}-\nu_{y, s}=p .
\end{aligned}
$$


The $A_{\tau}$ are assumed to be small compared to $A_{s}$, and the $B_{r}$ small compared to $B_{s} . \nu_{x, s_{3}}$ $\nu_{y, s}$ will give the $\nu$-values of the normal modes. The normal mode $\nu$-values are $\nu_{1}, \nu_{2}$ and we assume $\nu_{1} \rightarrow \nu_{x}$ and $\nu_{2} \rightarrow \nu_{y}$ when $a_{1} \rightarrow 0$, then $\nu_{x, s} \rightarrow \nu_{x}$ for the $\nu_{1}$ mode, and $\nu_{y, s} \rightarrow \nu_{y}$ for the $\nu_{2}$ mode, when $a_{1} \rightarrow 0$. The justification for choosing this form for the solutions, and the choice of the $\nu_{x, r}$ and the $\nu_{y, r}$ present will come out of the solution one finds using this form.

The $\nu_{x, r}$ and $\nu_{y, r}$ for $r \neq s$ will be seen to have the form

$$
\begin{aligned}
& \nu_{x, r}=\nu_{x, s}+n \\
& \nu_{y, r}=\nu_{y, s}+m
\end{aligned}
$$

where $n, m$ are integers. This could be assumed from the beginning. An alternative procedure is not to restrict $\nu_{x, r}$ and $\nu_{y, r}$, and to make the $\exp \left(i \nu_{x, r} \theta_{x}\right)$ an orthogonal set by choosing $\nu_{x, r}=(2 \pi / T) q, q$ is some integer and $T$ is some very large angle, and treating $\nu_{y, r}$ similarly. Putting Eq. (2.4) into Eq. (2.1) and using the orthogonal property, one finds

$$
\begin{aligned}
& \left(\nu_{x, r}^{2}-\nu_{x}^{2}\right) A_{r}=-2 \nu_{x} \sum_{r^{\prime}} b_{x}\left(\nu_{x, r}, \nu_{y, r}\right) B_{r} \\
& \left(\nu_{y, r}^{2}-\nu_{y}^{2}\right) B_{r}=-2 \nu_{y} \sum_{r^{\prime}} b_{y}\left(\nu_{y, r}, \nu_{x, r^{\prime}}\right) A_{r^{\prime}} \\
& b_{x}\left(\nu_{x, r}, \nu_{y, r^{\prime}}\right)=\frac{1}{2 T} \int_{0}^{T} d \theta_{x} \beta_{x}\left(\beta_{x} \beta_{y}\right)^{\frac{1}{2}}\left(a_{1} / \rho\right) \exp \left[i\left(-\nu_{x, r} \theta_{x}+\nu_{y, r^{\prime}} \theta_{y}\right)\right], \\
& b_{y}\left(\nu_{y, r}, \nu_{x, r^{\prime}}\right)=\frac{1}{2 T} \int_{0}^{T} d \theta_{y} \beta_{y}\left(\beta_{x} \beta_{y}\right)^{\frac{1}{2}}\left(a_{1} / \rho\right) \exp \left[i\left(-\nu_{y, r} \theta_{y}+\nu_{x, r^{\prime}} \theta_{x}\right)\right] .
\end{aligned}
$$

In Eq. (2.6) we assume $B_{r}<<B_{s}, A_{r}<<A_{s}$ for $r \neq s$ and find the first order results

$$
\begin{aligned}
& \left(\nu_{x, s}^{2}-\nu_{x}^{2}\right) A_{s}=-2 \nu_{x} b_{x}\left(\nu_{x, s}, \nu_{y, s}\right) B_{s} \\
& \left(\nu_{y, s}^{2}-\nu_{y}^{2}\right) B_{s}=-2 \nu_{y} b_{y}\left(\nu_{y, s}, \nu_{x, s}\right) A_{s} \\
& \left(\nu_{x, r}^{2}-\nu_{x}^{2}\right) A_{r}=-2 \nu_{x} b_{x}\left(\nu_{x, r}, \nu_{y, s}\right) B_{s} \\
& \left(\nu_{y, r}^{2}-\nu_{y}^{2}\right) B_{r}=-2 \nu_{y} b_{y}\left(\nu_{y, r}, \nu_{x, s}\right) A_{s}
\end{aligned}
$$

The first two equations in Eq. (2.7) are homogeneous equations for $A_{s}$ and $B_{s}$, and the $\nu$-values $\nu_{x, s}, \nu_{y, s}$ are determined by requiring the matrix of the coefficients of $A_{s}, B_{s}$ 
to vanish. This gives

$$
\begin{aligned}
& \left(\nu_{x, s}^{2}-\nu_{x}^{2}\right)\left(\nu_{y, s}^{2}-\nu_{y}^{2}\right)=4 \nu_{x} \nu_{y}\left|\Delta \nu\left(\nu_{x, s}, \nu_{y, s}\right)\right|^{2} \\
& \Delta \nu\left(\nu_{x, s}, \nu_{y, s}\right)=\frac{1}{4 \pi} \int_{0}^{2 \pi} d s\left(\beta_{x}, \beta_{y}\right)^{\frac{1}{2}}\left(a_{1} / \rho\right) \exp \left[i\left(-\nu_{x, s} \theta_{x}+\nu_{y, s} \theta_{y}\right)\right] \\
& \nu_{x, s}-\nu_{y, s}=p
\end{aligned}
$$

Eq. (2.8) can be simplified by assuming that $\nu_{x}, \nu_{y}$ are close to the resonance line $\nu_{x, s}-$ $\nu_{y, s}=p$ and $\nu_{x, s} \simeq \nu_{x}$ and $\nu_{y, s} \simeq \nu_{y}$. Keeping terms of lowest order only, one gets

$$
\begin{aligned}
& \left(\nu_{x, s}-\nu_{x}\right)\left(\nu_{y, s}-\nu_{y}\right)=\left|\Delta \nu\left(\nu_{x, s}, \nu_{y, s}\right)\right|^{2} \\
& \nu_{x, s}-\nu_{y, s}=p
\end{aligned}
$$

Eq. (2.9) has two solutions for $\nu_{x, s}, \nu_{y, s}$. We denote by $\nu_{1}$ the value of $\nu_{x, s}$ that goes to $\nu_{x}$ when $a_{1} \rightarrow 0$, and $\nu_{2}$ the value of $\nu_{y, s}$ that goes to $\nu_{y}$ when $a_{1} \rightarrow 0$. The solutions can be written as

$$
\begin{aligned}
& \nu_{1}=\bar{\nu}_{x} \pm\left\{\left(\frac{\nu_{x}-\nu_{y}-p}{2}\right)^{2}+\left|\Delta \nu\left(\bar{\nu}_{x}, \bar{\nu}_{y}\right)\right|^{2}\right\}^{\frac{1}{2}}, \\
& \nu_{2}=\bar{\nu}_{y} \mp\left\{\left(\frac{\nu_{x}-\nu_{y}-p}{2}\right)^{2}+\left|\Delta \nu\left(\bar{\nu}_{x}, \bar{\nu}_{y}\right)\right|^{2}\right\}^{\frac{1}{2}} \\
& \bar{\nu}_{x}=\left(\nu_{x}+\nu_{y}+p\right) / 2, \bar{\nu}_{y}=\left(\nu_{y}+\nu_{x}-p\right) / 2
\end{aligned}
$$

For the \pm , the $+\operatorname{sign}$ is used when $\nu_{x}>\nu_{y}+p$ for $\nu_{1}$ and the opposite sign for $\nu_{2}$. In $\Delta \nu\left(\nu_{x, s}, \nu_{y, s}\right), \nu_{x, s}$ has been replaced by $\bar{\nu}_{x}$, and $\nu_{y, s}$ by $\bar{\nu}_{y}$, which introduces a higher order error that can be neglected.

From Eq. (2.10) one finds

$$
\begin{aligned}
\left|\nu_{1}-\nu_{2}-p\right| & =2\left\{\left(\frac{\nu_{x}-\nu_{y}-p}{2}\right)^{2}+\left|\Delta \nu\left(\bar{\nu}_{x}, \bar{\nu}_{y}\right)\right|^{2}\right\}^{\frac{1}{2}} \\
\nu_{1}+\nu_{2} & =\nu_{x}+\nu_{y}
\end{aligned}
$$




\section{Higher Order Shifts in $\nu_{1}$ and $\nu_{2}$}

To find a higher order result for $\nu_{1}$ and $\nu_{2}$, one has to find higher order equations for $A_{s}, B_{s}$ by putting the lower order solution for $A_{r}, B_{r}, r \neq s$, given by Eq. (2.7) into Eq. (2.6).

Eq. (2.7) for $A_{r}, B_{r}$ can be somewhat simplified by assuming that $\nu_{x}, \nu_{y}$ are close to the resonance line $\nu_{x, s}=\nu_{y, s}+p$ so that one can assume that $\nu_{x, s} \simeq \nu_{x}$ and $\nu_{y, s} \simeq \nu_{y}$ and then

$$
\begin{aligned}
& A_{r}=\frac{-2 \nu_{x} b_{x}\left(\nu_{x, r}, \nu_{y, s}\right)}{\left(n+\nu_{x}+\nu_{y}\right)(n-p)} B_{s}, n \neq p \\
& B_{r}=\frac{-2 \nu_{y} b_{y}\left(\nu_{y, r}, \nu_{x, s}\right)}{\left(n+\nu_{x}+\nu_{y}\right)(n+p)} A_{s}, n \neq-p
\end{aligned}
$$

where $\nu_{x, r}=\nu_{y, s}+n$ and $\nu_{y, r}=\nu_{x, s}+n$.

Putting these results for $A_{\mathrm{r}}, B_{\mathrm{r}}$ in Eq. (2.6) one finds the improved equations for $A_{s}, B_{s}$

$$
\begin{aligned}
& \left(\nu_{x, s}^{2}-\nu_{x}^{2}-\Delta_{x}\right) A_{s}=-2 \nu_{x} b_{x}\left(\nu_{x, s}, \nu_{y, s}\right) B_{s}, \\
& \left(\nu_{y, s}^{2}-\nu_{y}^{2}-\Delta_{y}\right) B_{s}=-2 \nu_{y} b_{y}\left(\nu_{y, s}, \nu_{x, s}\right) A_{s} . \\
\Delta_{x}= & 4 \nu_{x} \nu_{y} \sum_{n \neq p} \frac{\left|c_{n}\right|^{2}}{\left(n-\nu_{x}-\nu_{y}\right)(n-p)}, \\
\Delta_{y}= & 4 \nu_{x} \nu_{y} \sum_{n \neq-p} \frac{\left|b_{n}\right|^{2}}{\left(n-\nu_{x}-\nu_{y}\right)(n+p)} \\
b_{n}= & \frac{1}{4 \pi \rho} \int d s a_{1}\left(\beta_{x} \beta_{y}\right)^{\frac{1}{2}} \exp \left[i\left(\left(n-\nu_{y}\right) \theta_{x}+\nu_{y} \theta_{y}\right)\right] \\
c_{n}= & \frac{1}{4 \pi \rho} \int d s a_{1}\left(\beta_{x} \beta_{y}\right)^{\frac{1}{2}} \exp \left[i\left(\left(n-\nu_{x}\right) \theta_{y}+\nu_{x} \theta_{x}\right)\right]
\end{aligned}
$$

Eq. (3.2) gives the equation for $\nu_{x, s}$ and $\nu_{y, s}$

$$
\begin{aligned}
& \left(\nu_{x, s}^{2}-\nu_{x}^{2}-\Delta_{x}\right)\left(\nu_{y, s}^{2}-\nu_{y}^{2}-\Delta_{y}\right)=4 \nu_{x} \nu_{y}\left|\Delta \nu\left(\nu_{x, s}, \nu_{y, s}\right)\right|^{2} \\
& \nu_{x, s}=\nu_{y, s}+p
\end{aligned}
$$

Eq. (3.3) was obtained by using the result for $A_{r}, B_{r}$ which is first order in $a_{1}$. By iterating Eq. (2.6) one can find a result for $A_{r}, B_{r}$ to second order in $a_{1}$ which will change Eq. (3.3) by replacing $\Delta \nu$ by

$$
\Delta \nu \rightarrow \Delta \nu+\Delta \nu^{(3)}
$$


where $\Delta \nu^{(3)}$ is third order in $a_{1}$. By going one step further and iterating Eq. (2.6) to find results for $A_{r}, B_{r}$ to third order in $a_{1}$ will change Eq. (3.3) by replacing $\Delta_{x}, \Delta_{y}$ by

$$
\begin{aligned}
& \Delta_{x} \rightarrow \Delta_{x}+\Delta_{x}^{(4)} \\
& \Delta_{y} \rightarrow \Delta_{y}+\Delta_{y}^{(4)}
\end{aligned}
$$

where $\Delta_{x}^{(4)}, \Delta_{y}^{(4)}$ are fourth order in $a_{1}$. One can write down all these higher order terms. However, the expression Eq. (3.3) keeping terms up to second order in $a_{1}$ is probably sufficient here.

One should also note that in Eq. (3.3) $\nu_{x, s}$ and $\nu_{y, s}$ also occur implicitly in $\Delta \nu\left(\nu_{x, s}, \nu_{y, s}\right)$ which complicates the solution of Eq. (3.3) for $\nu_{x, s}$ and $\nu_{y, s}$. Solutions can be found depending on the size of $\Delta \nu$ and the distance from the resonance line $\nu_{x}=\nu_{y}+p$.

One interesting case is when a 2 family $a_{1}$ correction system is used to make $\Delta \nu=0$, and when $\nu_{x}, \nu_{y}$ are very close to the resonance line $\nu_{x}-\nu_{y}=p$, so that $\nu_{1}=\nu_{x}$ and $\nu_{2}=\nu_{y}$ with an error that is second order in $a_{1}$. Very close to the resonance line, so that in Eq. (2.10) $\left(\nu_{x}-\nu_{y}-p\right)^{2} / 4$ can be neglected compared to $|\Delta \nu|^{2}$, then the above can be achieved by making $\Delta \nu\left(\bar{\nu}_{x}, \bar{\nu}_{y}\right)=0$ as shown in Eq. (2.10).

This corresponds roughly to the situation when a 2 family $a_{1}$ correction is used to cancel the driving term of the nearby difference resonance, $\nu_{x}-\nu_{y}=p$. In this situation, one can find the shift in $\nu_{x, s}$ and $\nu_{y, s}$ due to the second order $\Delta_{x}, \Delta_{y}$. Then in Eq. (3.3) $\Delta \nu\left(\nu_{x, s}, \nu_{y, s}\right)$ is not zero but differs from zero by terms of order $a_{1}^{3}$, and thus $|\Delta \nu|^{2}$ is of order $a_{1}^{6}$. For this result, the previous observation, that higher order terms can only change the $\Delta \nu$ term by $\Delta \nu^{(3)}$, a term of third order, is significant. As $|\Delta \nu|^{2}$ is of order $a_{1}^{6}$, one can treat it as being zero, and Eq. (3.3) becomes

$$
\left(\nu_{x, s}^{2}-\nu_{x}^{2}-\Delta_{x}\right)\left(\nu_{y, s}^{2}-\nu_{y}^{2}-\Delta_{y}\right)=0
$$

which gives the normal modes

$$
\begin{aligned}
& \nu_{1}=\nu_{x}+\frac{1}{2 \nu_{x}} \Delta_{x} \\
& \nu_{2}=\nu_{y}+\frac{1}{2 \nu_{y}} \Delta_{y} .
\end{aligned}
$$

Thus for the case when $\Delta \nu=0$ and close to the resonance line, there is a second order in $a_{1}$ shift in the $\nu$-values given by $\Delta_{x} / 2 \nu_{x}$ and $\Delta_{y} / 2 \nu_{y}$. Eq. (3.2) for $\Delta_{x}$ and $\Delta_{y}$ show 
that the largest second order $\nu$-shifts will come from harmonics in $a_{1}$ close to $\nu_{x}+\nu_{y}$. The driving terms $b_{n}$ and $c_{n}$ for $n$ closest to $\nu_{x}+\nu_{y}$ contribute mosi to the second order $\nu$-shifts.

One may also notice that $b_{n}, c_{n}$, as given by Eq. (3.2), are just the usual stop-band results for the $\nu_{x}+\nu_{y}=n$ resonance but evaluated at particular points on the resonance line. $b_{n}$ corresponds to the point $n-\nu_{y}, \nu_{y}$ and $c_{n}$ to the point $\nu_{x}, n-\nu_{x}$. For the $n$-values corresponding to resonance lines closest to the unperturbed $\nu_{x}, \nu_{y}$, these points on the resonance are not far apart and the $b_{n}$ and $c_{n}$ are about equal. Thus for the $\nu_{x}+\nu_{y}=n$ lines closest to the unperturbed $\nu_{x}, \nu_{y}, \nu_{1}$ and $\nu_{2}$ are shifted about equally and these $b_{n}$, $c_{n}$ do not contribute much to the residual $\left|\nu_{1}-\nu_{2}\right|$. This lack of a dominant harmonic for the residual $\left|\nu_{1}-\nu_{2}\right|$ makes the correction of the residual $\left|\nu_{1}-\nu_{2}\right|$ more difficult.

Eq. (3.7) has been checked ${ }^{4}$ by comparing these results with numerical computations of $\nu_{1}, \nu_{2}$. For the case of $\nu_{x}=\nu_{y}$ resonance line, $p=0$, Eq. (3.3) may be solved for $\nu_{x, s}$, $\nu_{y, s}$ and written as

$$
\begin{aligned}
& \nu_{1}=\frac{1}{2}\left(\tilde{\nu}_{x}^{2}+\tilde{\nu}_{y}^{2}\right) \pm\left\{\left(\frac{\tilde{\nu}_{x}^{2}-\tilde{\nu}_{y}^{2}}{2}\right)^{2}+4 \nu_{x} \nu_{y}\left|\Delta \nu\left(\nu_{1}, \nu_{1}\right)\right|^{2}\right\}^{\frac{1}{2}} \\
& \nu_{2}=\frac{1}{2}\left(\tilde{\nu}_{x}^{2}+\tilde{\nu}_{y}^{2}\right) \mp\left\{\left(\frac{\tilde{\nu}_{x}-\nu_{y}^{2}}{2}\right)^{2}+4 \nu_{x} \nu_{y}\left|\Delta \nu\left(\nu_{2}, \nu_{2}\right)\right|^{2}\right\}^{\frac{1}{2}} \\
& \tilde{\nu}_{x}^{2}=\nu_{x}^{2}+\Delta_{x}, \quad \tilde{\nu}_{y}^{2}=\tilde{\nu}_{y}^{2}+\Delta_{y}
\end{aligned}
$$

$\nu_{1}$ is the mode that goes to $\nu_{x}$ when $a_{1} \rightarrow 0$, and $\nu_{2}$ goes to $\nu_{y}$. For the \pm sign, the + sign is used when $\nu_{x}>\nu_{y}$ for $\nu_{1}$ and the opposite sign for $\nu_{2}$. One can derive Eq. (3.7) from Eq. (3.8) when $\Delta \nu(\bar{\nu}, \bar{\nu})=0, \bar{\nu}=\frac{1}{2}\left(\nu_{x}+\nu_{y}\right)$, and close to the resonance line $\nu_{x}=\nu_{y}$. 


\section{4. $\nu$-Shifts when $\nu_{x}, \nu_{y}$ are far from the $\nu_{x}-\nu_{y}=p$ Resonance}

In the derivation of the previous results, $\nu_{x}, \nu_{y}$ were assumed to be close to the $\nu_{x}-\nu_{y}=$ $p$ resonance line. When $\nu_{x}, \nu_{y}$ are far from the resonance line the results are less interesting as the $\nu$-shifts are of higher order and smaller. However, it is interesting to see how the results for the $\nu$ shifts in these two cases will fit together.

Up to Eq. (2.6), the previous derivation will hold when $\nu_{x}, \nu_{y}$ are far from the $\nu_{x}-\nu_{y}=$ $p$ resonance line. Let us first consider the $\nu_{1}$ mode where $\nu_{1} \rightarrow \nu_{x}$ when $a_{1} \rightarrow 0$. In this case, it is assumed that not only the $A_{\tau}$ are small compared to $A_{s}$, but also $B_{s}$ is small.

To lowest order, Eq. (2.7) become

$$
\begin{aligned}
\left(\nu_{x, s}^{2}-\nu_{x}^{2}\right) A_{s} & =0 \\
\left(\nu_{x, r}^{2}-\nu_{x}^{2}\right) A_{r} & =0 \\
\left(\nu_{x, r}^{2}-\nu_{y}^{2}\right) B_{r} & =-2 \nu_{y} b_{y}\left(\nu_{y, r}, \nu_{x, s}\right) A_{s} \\
\nu_{y, r} & =\nu_{x, s}+n .
\end{aligned}
$$

Thus to lowest order, $\nu_{1}=\nu_{x}$, and the tune shift is a higher order effect in $a_{1}$. To find the second order shift in $\nu_{1}$, the result for $B_{\tau}$ in Eq. (4.1) is put into Eq. (2.6) and the $A_{s}$ equation becomes

$$
\begin{aligned}
\left(\nu_{x, s}^{2}-\nu_{x}^{2}\right) A_{s} & =\bar{\Delta}_{x} A_{s} \\
\bar{\Delta}_{x} & =4 \nu_{x} \nu_{y} \sum_{n} \frac{\left|c_{n}\right|^{2}}{\left(n-\nu_{x}\right)^{2}-\nu_{y}^{2}} \\
c_{n} & =\frac{1}{4 \pi \rho} \int d s a_{1}\left(\beta_{x} \beta_{y}\right)^{\frac{1}{2}} \exp \left[i\left(\left(n-\nu_{x}\right) \theta_{y}+\nu_{x} \theta_{x}\right)\right] .
\end{aligned}
$$

This gives the shift in $\nu_{x}$,

$$
\nu_{1}^{2}=\nu_{x}^{2}+\bar{\Delta}_{x}
$$

The $\bar{\Delta}_{x}$ is similar to the $\Delta_{x}$ in Eq. (3.2) except that we now do not assume that $\nu_{x}-\nu_{y} \cong p$ and the sum over $n$ is over all $n$. This result, Eq. (4.2b), can be obtained from Eq. (3.3) if in Eq. (3.3) we assume that

$$
\left(\nu_{y, s}^{2}-\nu_{y}^{2}-\Delta_{y}\right) \simeq\left(\left(\nu_{x}-p\right)^{2}-\nu_{y}^{2}\right),
$$

and not replace $\nu_{x}-\nu_{y}$ by $p$ in Eq. (3.2) for $\Delta_{x}$. 
In the same way one finds for the $\nu_{2}$ mode,

$$
\begin{aligned}
\nu_{2}^{2} & =\nu_{y}^{2}+\bar{\Delta}_{y} \\
\bar{\Delta}_{y} & =4 \nu_{x} \nu_{y} \sum_{n} \frac{\left|b_{n}\right|^{2}}{\left(n-\nu_{y}\right)^{2}-\nu_{x}^{2}} \\
b_{n} & =\frac{1}{4 \pi \rho} \int d s a_{1}\left(\beta_{x} \beta_{y}\right)^{\frac{1}{2}} \exp \left[i\left(\left(n-\nu_{y}\right) \theta_{x}+\nu_{y} \theta_{y}\right)\right] .
\end{aligned}
$$

\section{References}

1. E.D. Courant and H.S. Snyder, Ann. Phys. 3, 1 (1958).

2. D. Edwards and L. Teng, IEEE 1973 PAC, p. 885 (1973).

3. G. Parzen, IEEE 1991 PAC (1991), also BNL Report AD/RHIC-82 (1990).

4. G. Parzen, BNL Report AD/RHIC/AP-93 (1991). 